\title{
A Projector-Camera System for Creating a Display with Water Drops
}

\author{
Peter Barnum
}

\author{
Srinivasa Narasimhan \\ Carnegie Mellon University \\ Pittsburgh, PA, USA \\ \{pbarnum, srinivas, tk\}@cs.cmu.edu
}

\author{
Takeo Kanade
}

\begin{abstract}
Various non-traditional media, such as water drops, mist, and fire, have been used to create vibrant two and three dimensional displays. Usually such displays require a great deal of design and engineering. In this work, we show a computer vision based approach to easily calibrate and learn the properties of a three-dimensional water drop display, using a few pieces of off-the-shelf hardware.

Our setup consists of a camera, projector, laser plane, and water drop generator. Based on the geometric calibration between the hardware, a user can "paint" the drops from the point of view of the camera, causing the projector to illuminate them with the correct color at the correct time. We first demonstrate an algorithm for the case where no drop occludes another from the point of view of either camera or projector. If there is no occlusion, the system can be trained once, and the projector plays a precomputed movie. We then show our work toward a display with real rain. In real time, our system tracks and predicts the future location of hundreds of drops per second, then projects rays to hit or miss each drop.
\end{abstract}

\section{Introduction and previous work}

Traditional displays, such as CRT and LCD screens, are compact packages used to deliver two dimensional images on a glass or plastic screen. Non-traditional displays use unusual media, such as fog, fire, or water drops, as carriers of two or three dimensional information.

Some displays create a picture by controlling the medium. These displays require large banks of valves controlled with high speed and precision. Examples of these use falling drops [13, 15], bubbles [7], and fire [11]. (A good review of other types is presented in [9]). In these cases, a constant light illuminates the apparatus, which emits particles in a controlled fashion. These particles act as voxels. For example, a large water drop will act as a bright

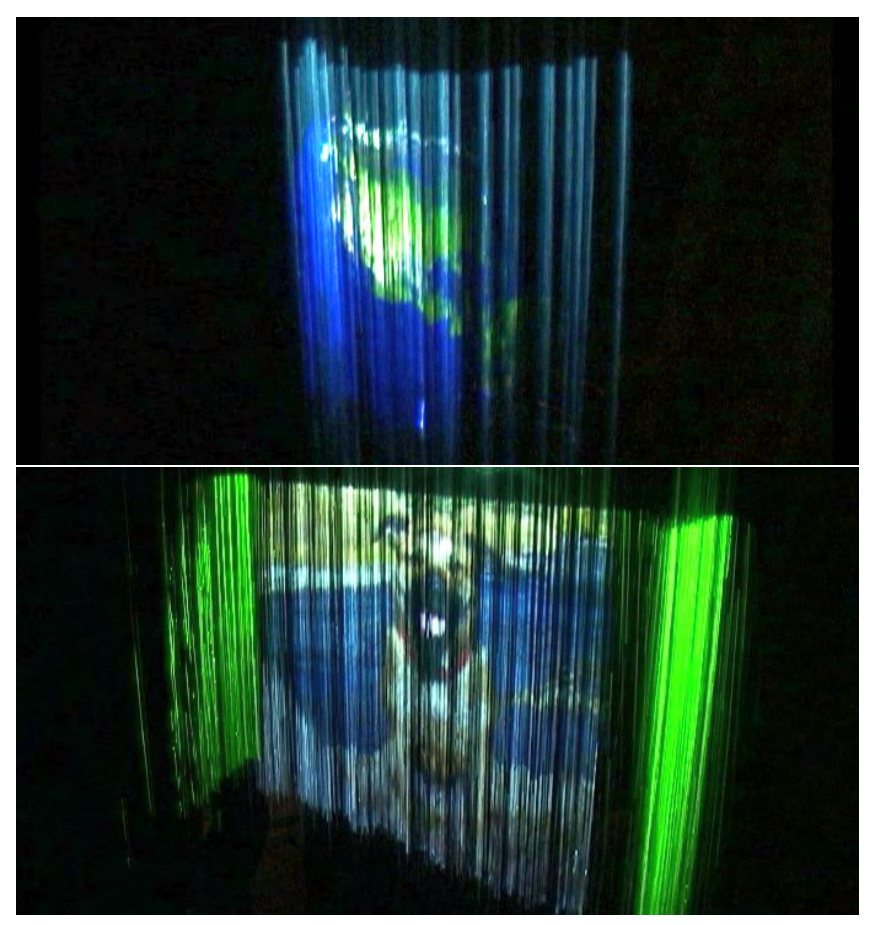

Figure 1. Two examples of three-dimensional water displays. The spinning globe is projected on a cylinder of water streams. The dog is projected on a flat plane surrounded by green walls.

voxel, while collections of small water drops create many dim voxels.

Alternately, the display can be created by controlling the illumination. In order for the displays to be coherent, these methods involve precise design of the drop or fog emitters, to insure that they create predictable and constant streams. Some designs focus on abstract patterns. The water drop display by Eitoku et al. [4] makes colorful three-dimensional patterns with a projector and falling water drops. Other designs emulate traditional displays, such as flat fog screens $([1,12]$ and others).

As demonstrated in the above examples, non-traditional 


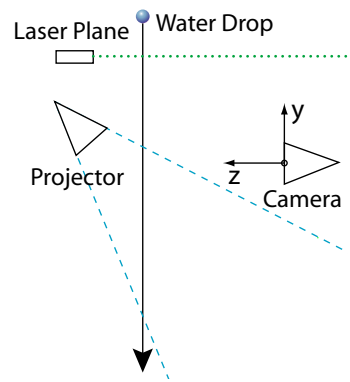

(a)

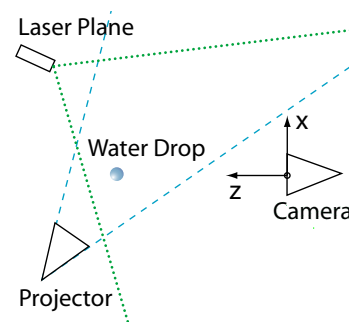

(b)

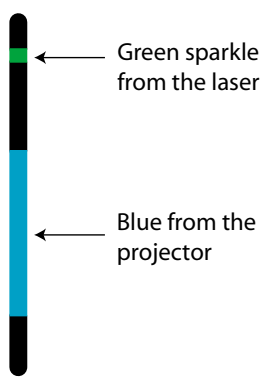

(c)

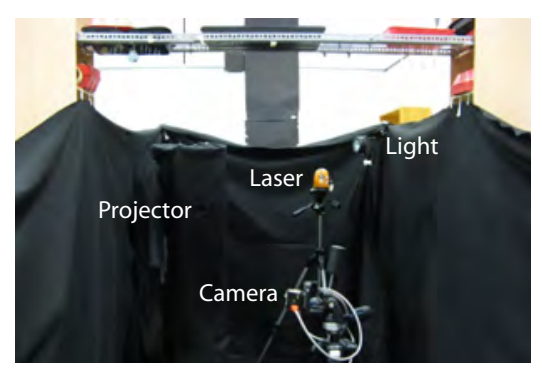

(d)

Figure 2. A side view (a) and top view (b) of a water drop as it crosses the laser plane and projector field of view. The image generated is shown in (c), and a photograph of the setup in (d). All of our equipment is standard off-the-shelf hardware. The projector is a 3LCD Panasonic PT-L701U, which can run between $60-85 \mathrm{hz}$ at its native resolution of $1024 \times 768$. The laser plane is a AccuLine Pro 40-6640, which is a class IIIa laser level. The camera is a Point Grey Firefly MV that captures 60 uncompressed frames per second with a $752 x 480$ Bayer array. It can run faster if fewer consecutive rows of pixels are sampled, such as $111 \mathrm{FPS}$ at $752 \times 240$. The drop generator is a styrofoam or plastic box with plastic pipette tips or small holes as water emitters.

displays usually involve precise engineering and equipment. But in this work, we demonstrate a computer-vision based method to calibrate and learn the properties of a water display, using only a few pieces of off-the-shelf hardware. With our method, a three-dimensional display can be created with calibration and manipulation in image coordinates, without the need for explicit 3D reasoning.

The display is created by a projector that illuminates water drops falling from a drop generator. Figure 2 shows an example of one drop from one emitter. The threedimensional position of the drop is determined based on the image location where it intersects the laser plane. (Our depth and velocity measuring technique bears similarity to optical distrometers $[16,8]$, but can handle dynamics greater than first-order).

We calibrate geometrically by creating a lookup table of homographies between the camera and projector, based on the image location of a water drop's laser plane intersection. Using this lookup, we can also predict the drop's future projector coordinates based only on its image locations. The drops can therefore be intuitively "painted" in the camera's reference frame.

We first examine the case where water drops never occlude each other from the point of view of either camera and projector. The detected drops are processed offline, then the projector plays a precomputed movie. However, if drops can occlude each other, as in real rain, then the projector must display different patterns for different drop locations and times. This requires the real-time tracking and prediction of the future locations of hundreds of drops per second.

\section{A 3D display in a controlled environment}

In this section, we describe how to create a 3D animated display with a camera, projector, laser plane, and water drop generator. Figure 2 (d) is a photograph of the setup from slightly behind the camera. In this section, we require that the drop generator is designed so that no drop occludes another from the point of view of the camera or the projector. This means that once we determine which projector pixels illuminate which drops, the camera does not have to be observe the scene. The display is animated by computing a movie made of the geometrically calibrated images, then projecting the movie on the drops.

Creating a 3D display (or any 3D model) involves two steps. First, the shape of the model must be designed. This could be a polygon mesh or a voxel occupancy grid. For our setup, this means that the artist must decide where to punch holes in the drop generator. The locations of the holes determines where the drops fall in the environment, which is the three dimensional shape of the water canvas. Second, the canvas is painted with a static or moving image.

Figure 2 shows an example of a single falling water drop. When it hits the laser plane, it creates a bright sparkle. As it continues to fall, it is illuminated by the projector. Because of the finite exposure time of the camera, it will appear as a motion-blurred streak in each image. One streak will have a bright green dot where the drop crosses the laser. An exact correspondence between the image, projector, and drop can be obtained by a one-time geometric calibration and the image location of the sparkle and the drop.

\subsection{Geometric calibration}

The key advantage of our method is that knowing the explicit 3D coordinates of each drop is unnecessary. The artist will paint the drops from the point of view of the camera, so all that is required is a mapping between image location of each drop and the projector location that will illuminate it. This mapping is an eight degrees of freedom linear transformation called a homography. The homography is not 
the same for every drop, but the correct homography can be computed based on the location of the sparkle where the drop crossed the laser plane.

A rear-projection screen is used for calibration. If we place the screen at depth $z$, parallel to the image plane shown in Figure 2, then anything projected will be imaged by the camera. Since the screen is kept parallel to the image plane, the image captured depends only on the screen's depth $z$. We define $\Pi_{z}$ as the plane at $z$.

Because the screen is parallel to the image plane and perpendicular to the laser plane, the laser plane is imaged as a horizontal line at height $y$. Due to perspective projection, the line has a unique $y$ location for each depth $z$. For a plane $\Pi_{z}$ that creates a laser line at $y$, there is a homography $H_{y}$ that warps the image coordinates to the projector coordinates.

The next step is to create a lookup table for $H_{y}$. We project a black and white checkerboard pattern, and acquire a video as the rear projector screen is moved along the $z$ axis. The $y$ location of the green line in each image is computed as the maximum row of a one-channel image of green $-($ red + blue $)$. For one reference image with laserline location $r$, we compute the camera-projector homography $H_{r}$ by hand-labeling point correspondences between the image and the checkerboard that was projected. We then use the non-pyramidal OpenCV feature point tracker to find correspondences between the reference image and all other images. Based on these point correspondences, we compute the homography $H_{y \rightarrow r}$ between each image and the reference image. The homography for the line at that $y$ is then simply $H_{y}=H_{r} H_{y \rightarrow r}$.

Since the $z$ location of a falling drop does not vary greatly, knowing the $y$ location of its laser crossing and its image location is sufficient to determine the projector pixel that will illuminate it. Therefore, all tracking and inference will now be done completely in the image coordinates.

\subsection{Drop location calibration and display}

The next step is to find the drops' locations in the image. To determine the paths of the falling drops, we capture a few seconds of video and track each drop as it falls. In this section, drops do not occlude each other and tracking could be performed offline. However, they do need to be tracked in real time in the next section, and we use the same setup.

Falling drops create motion-blurred streaks in the images. We segment the streaks and track them by matching the endpoints of neighboring streaks across consecutive frames. The user tags the locations where drops from each generator cross the laser plane. All tracked drops are then assigned to the nearest tagged location. For each emitter, the drop that is tracked the longest is used as the estimate of the path of all drops.

Once drops from each emitter have been tracked, the wa- ter drops are "painted". The artist colors the model from the point of view of the camera. We developed a simple MATLAB design interface to allow even an inexperienced user to position and scale images and movies on the image. Once the artist has determined how the display should appear from the point of view of the camera, each image point is warped based on the homography for the closest drop, creating a movie for the projector to then display.

\subsection{Example displays}

We created several display geometries, shown in Figure 3. Each was calibrated using only a few drop emitters. To create a flow of approximately $10 \mathrm{drops} / \mathrm{sec}$, we used low binding $10 \mu l$ pipette tips for each emitter. Once calibrated, we drilled additional $2.38 \mathrm{~mm}$ holes, increasing the water flow to a stream. Placing additional holes and increasing the amount of water increases the display's brightness and resolution, but does not require additional calibration since the water covers the same calibrated planes. Specific details of each are discussed in the figure captions.

Many arrangements are possible, even with the geometry restrictions needed to prevent occlusion. The simplest is to arrange all emitters in a line, to create the flat water screen shown in Figure 3 (a).

Moving emitters forward and backward creates the Vshaped display in Figure 3 (b), which has one blue plane and one green plane. Because the shape is convex relative to the projector, many emitters can be placed in a small area without occluding each other. This allows for high horizontal resolution and large depth differences.

Horizontal resolution is traded for depth in the H-shaped display in Figure 3 (c). Unlike the previous example, parts of the three planes could occlude each other. If the three planes touched, some emitters would be in other emitters' shadows. This example illustrates the practical limits of drop generator design. As the complexity of the geometry increase, fewer emitters can be placed. In addition, due to the refraction of light within the water, the displays are brightest when viewed while facing the projector. Because of the large depth difference between parts of the display, the angle between viewer, water, and projector vary. This causes the right green plane to be brighter than the left.

The final example in Figure 3 (d) demonstrates a cylindrical screen generated by drop emitters arranged in a curve. We sample four locations along the curve, and fit thin-plate splines. One spline is used to determine the projector $(x, y)$ to camera $x$ mapping, and another for the projector $(x, y)$ to camera $y$ mapping. Although not as accurate as using a true 3D model, this allows us to display on a curved surface using the same algorithms as before, and without explicit geometric calibration. 


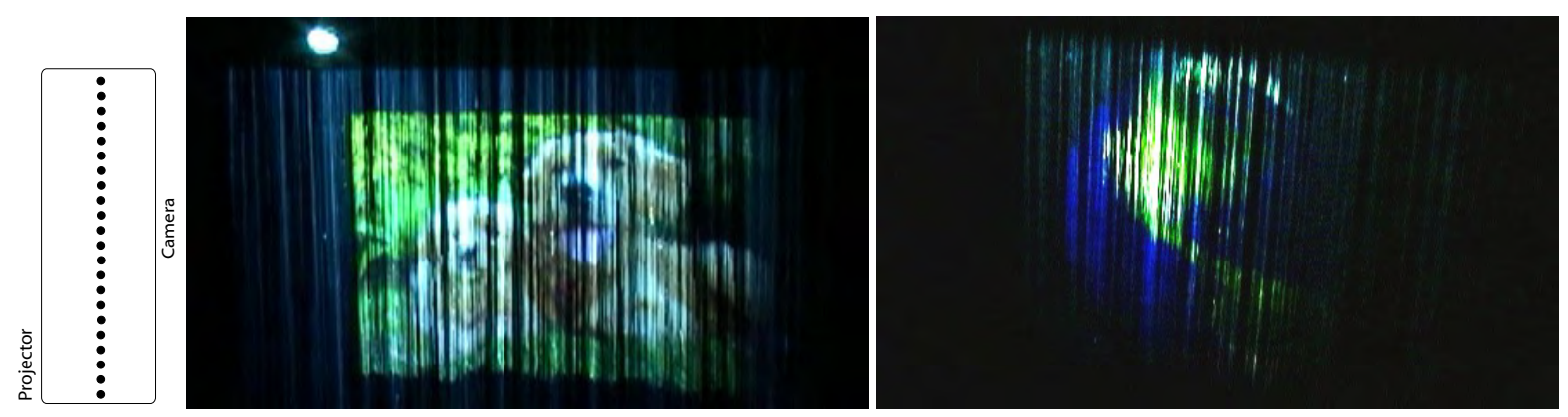

(a) Flat display: The flat display was calibrated with only one emitter at the center of the display. Once the homography for one drop traveling on a plane is known, the 70 additional emitters can be added on the same plane without additional calibration. Because the direction of the water streams vary a few degrees over time, some parts of the image are high resolution, while others parts have holes. As seen in our video, the holes' locations vary over time. The left image is from dailypuppy.com and the right from wikipedia.org.

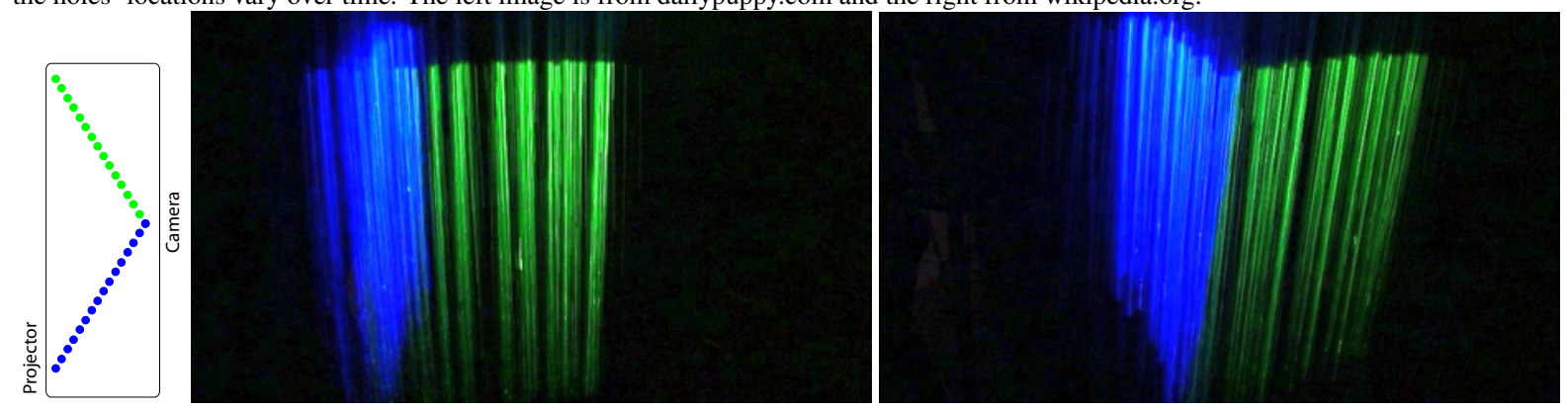

(b) V-shaped display: This display also required only one emitter to calibrate, at the intersection of the two planes. The 27 emitters to the left were colored blue and the 26 to the right were colored green.

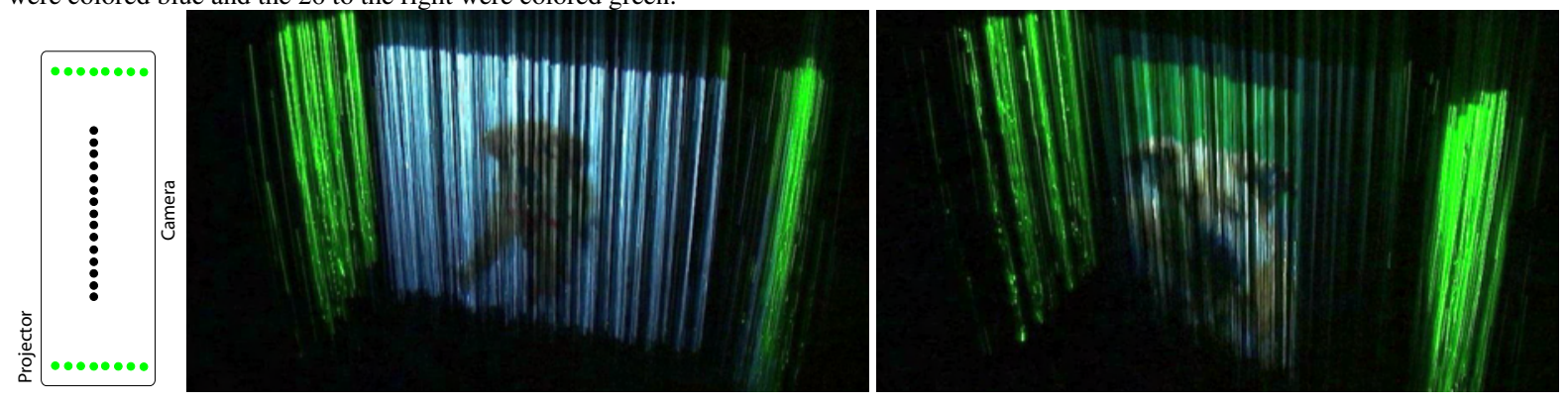

(c) $\mathrm{H}$-shaped display: Combining the high resolution display of the first example with the geometry of the second makes a 58 emitter flat display surrounded by 28 emitter walls. It required two emitters to calibrate, one on each side of the center panel. The images are from dailypuppy.com.
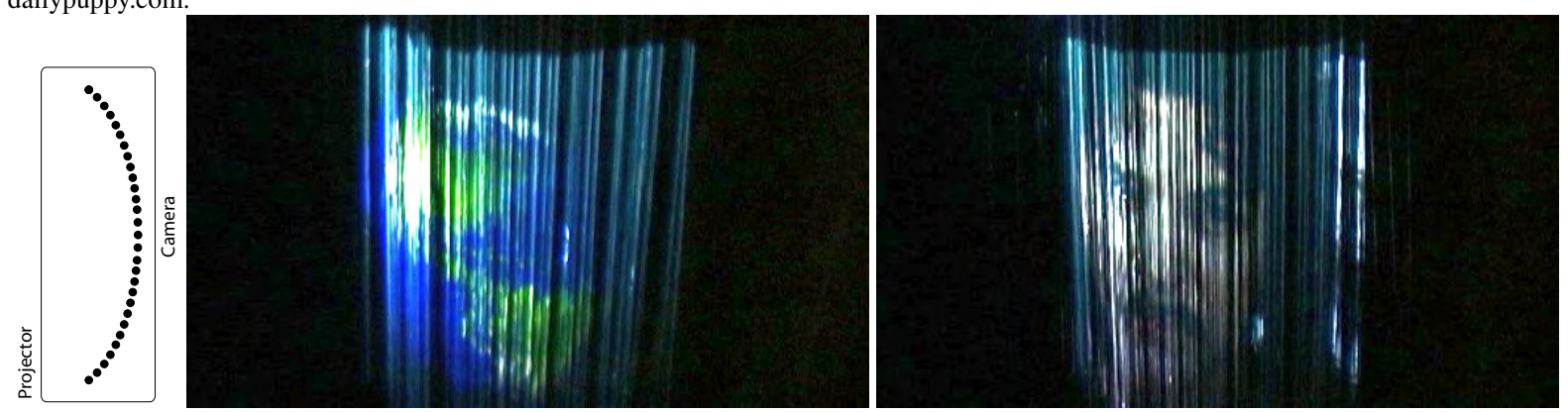

(d) Curved display: Four emitters and a thin-plate spline were used to calibrate this 41 emitter display. Any object that can be approximated by a vertically-constant curve can be projected with such a display, such as a globe or the face of Inigo Montoya from The Princess Bride.

Figure 3. Several example displays with different geometries. Each display was calibrated with a few emitters dropping individual water drops. Once calibrated, additional large emitters were added to create streams of water. 


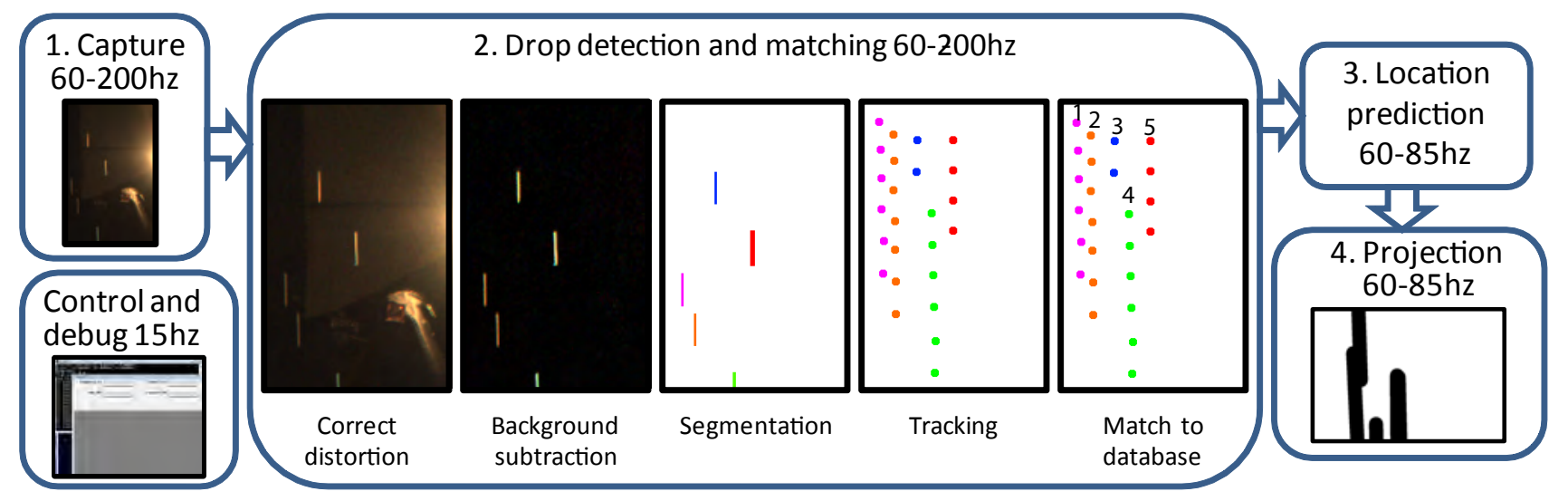

Figure 4. Control loop of the real-time tracking, prediction, and projection system. Each of the five bubbles represents an asynchronous loop, running at the given speed. Training ends after the fourth step of "Drop detection and tracking", while display uses all steps. For training, frames are captured at 60-200hz, then drops are segmented and tracked, and a database is created offline. Testing takes the tracks and matches them to the database to determine their future locations, then directs the projector to illuminate them. Control and debug displays recent image captures and sliders for background subtraction thresholds.
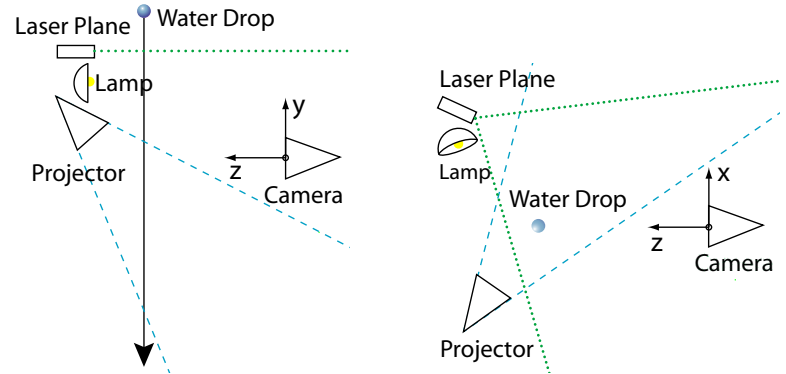

Figure 5. A side and top view of the drop-tracking setup. The lamp is roughly directional, but not to the same degree as a projector. It is bright enough to illuminate each drop along its entire path, but not so bright that it interferes with the display.

\section{Toward controlling illumination in real rain}

Even when no drop occludes another, interesting displays can be created. Creating exact drop-generator geometry is a valuable artistic tool, but one of our eventual goals is to create a display that can be used in real rain. In this section, we focus on algorithms and apparatus design appropriate to an unconstrained outdoor environment.

To display the correct image with the projector, we must detect and compute the trajectory of each drop before it is illuminated by the projector. Large raindrops can fall at over $9 \mathrm{~m} / \mathrm{s}$ [5], and even in light rain, a cubic meter contains hundreds of drops [14]. We demonstrate an algorithm that tracks and predicts the future location of hundreds of water drops per second, using the same camera and laser plane as the previous section.

A lamp is added to illuminate the drops between emis- sion and the projector, as shown in Figure 5. The algorithm still has geometric calibration, drop calibration, and display steps. But the drop calibration now involves learning the parameters of a model of the drops' dynamics. And unlike Section 2.2, projector pixels must be toggled based on the drops' locations.

Figure 4 outlines the complete algorithm for adaptive display. Each bubble represents an asynchronous loop. Step 1 is the camera capture loop. Depending on the requested resolution, the camera acquires frames between $60-200 \mathrm{hz}$. Step 2 is drop detection, tracking, and database matching. Training the database involves detecting and tracking drops for a few seconds, then building a prediction database offline. During display, detection and tracking are also performed, where each tracked drop is matched to the database. Once matched, Step 3 predicts each drop's future location and Step 4 projects the correct pattern.

\subsection{Streak detection and tracking}

Since the camera captures image at up to 200FPS, the entire process of segmentation and tracking must be completed within .005 seconds. Our approach is similar to raindrop tracking in [6], but is fast enough to work online. The first step is to correct for radial and tangential distortion. We precompute an integer-precision lookup table between distorted and undistorted pixels. The background subtraction is performed via absolute difference from a median image that is trained over several hundred frames. We perform approximate connected components [10] with a simplified version of raster-line based region growing [3]. For a common 752x480 image of streaks, connected components are found within .001 seconds on a single $3.2 \mathrm{Ghz}$ Xeon core. 
Once the images are segmented, tracking involves matching streak endpoints across frames. The camera shutter is left open, so the end of a streak in one frame will be in the same location as its beginning in the next.

\subsection{Drop dynamics calibration}

In order to determine which projector pixels to illuminate, we must predict each drop's image position $(x, y)$ at a future time $t$. Real raindrops have a constant velocity, so position can be predicted with a first order model. The drops from a drop generator fall slower, but they have more complex dynamics.

Drops are released from each emitter at slightly different initial velocities. The drops fall mostly straight down, from high to low $y$, and with a small change in $x$. The $x$ velocity varies and must be fit for each new drop. However, the $y$ position is determined by consistent factors, therefore it can be predicted with a model of drop dynamics.

Because of their complex, fluid shape, the drag on a drop is complex $[2,14]$, but it is normally consistent between different drops from the same emitter. As with Section 2.2, the user labels the intersection of each emitter with the laser plane. The dynamics model is then trained for each emitter. The top of each streak is the location of the drop at the beginning of the camera's exposure, and likewise for the bottom. A drop tracked for $N+1$ frames will have $N$ triplets, $\left(x_{0}, y_{0}, t_{0}\right), \ldots,\left(x_{N-1}, y_{N-1}, t_{N-1}\right)$, where $\frac{1}{t_{i}-t_{i-1}}$ equals the camera capture frequency.

We train on the longest track passing through each laserintersection sparkle. We subtract the starting time from all pairs, obtaining a sequence in the range $\left[0, t_{N-1}-t_{0}\right]$, then use robust linear least squares to learn a model of $y$ at time $t$ as a polynomial with three coefficients $a$ :

$$
y(t)=a_{0}+a_{1} t+a_{2} t^{2}
$$

\subsection{Adaptive projector control}

Matching a new query drop to the model is performed in a similar way to Section 2.2. A query drop is matched to its nearest neighbor in the database by finding the closest laser crossing. The future $x$ position is predicted with a firstorder polynomial fit. The future $y$ location is determined by fitting to the trained Equation 1.

First, the inverse of Equation 1 is used to find the value of $t$ that corresponds to each of the query drop's $y$ values, giving a set of $\left(y_{i}, t_{i}^{\prime}\right)$ pairs. If the query drop is falling at exactly the same rate as the trained model and estimation is perfect, then for a given camera frame rate $r$

$$
\forall i\left(t_{0}^{\prime}=t_{i}^{\prime}-i r\right)
$$

In practice, the estimates vary, so with $t_{0}^{*}$ being the mean of all $t_{0}^{\prime}$ estimates, the predicted location of the drop is

$$
y(t)=a_{0}+a_{1}\left(t-t_{0}+t_{0}^{*}\right)+a_{2}\left(t-t_{0}+t_{0}^{*}\right)^{2}
$$
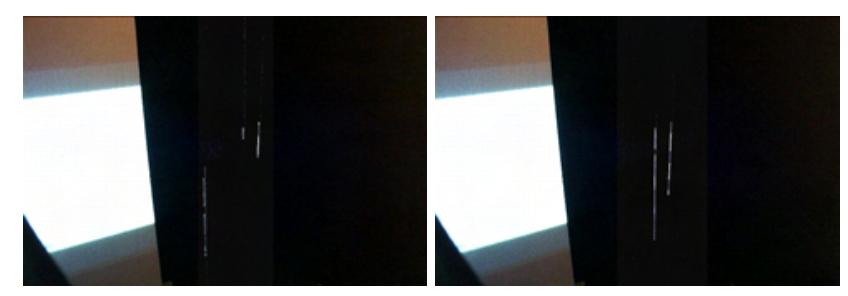

(a)
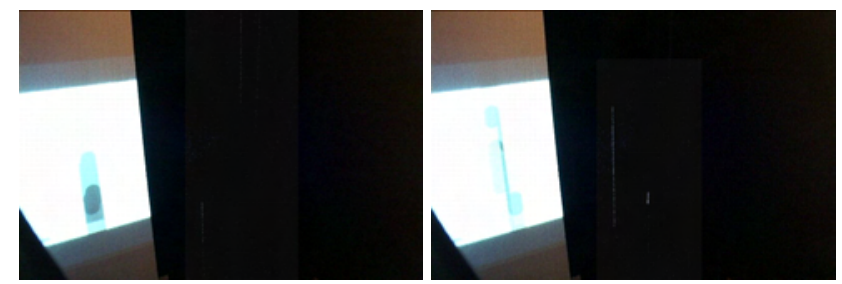

(b)

Figure 6. Selectively missing streaks. A screen is placed behind the drops, so the image projected can be viewed on the left and the effect on the drops on the right. Due to the low dynamic range of the camera, the streaks in the right half of each image have been brightened. (a) When the projector displays a white image, the streaks are visible. (b) But by tracking and selectively displaying black, the drops are not illuminated, so streaks are not visible.

Once the query drop is fit to the correct database drop, projector location prediction only requires the calibration from Section 2.1. For a given projector refresh frequency $f$, the drop will trace a line of image pixels over time $[t, t+$ $1 / f]$. The line in the image is then warped to the projector reference frame. The end result is that the projector will illuminate the drop when it passes in front.

\subsection{Results for real-time tracking and prediction}

Figure 6 shows an example demonstrating real time detection and projector control. We placed a screen behind the drops, so it is possible to see both the streaks and the image that was projected at that time. When the projector outputs a white image as in Figure 6 (a), the streaks can be seen. But since we know where the drop will be, we can project a black line, effectively "missing" the drop (Figure 6 (b)). This is the type of control that will be necessary for a display in real rain.

Because we track and predict drops in real time, we can create a display with less restriction on drop generator geometry. Figure 7 demonstrates an example with two rows of emitters that are occluded from the perspective of the projector. By tracking each drop, we can color the front row blue and the rear row red. Figure 7 (a) shows two frames where the tracking and projection is successful. Figure 7 (b) shows two failure cases. (The projector is running at $60 \mathrm{hz}$ and the camera at $15 \mathrm{hz}$, hence the four repetition of each streak). These errors occur because the projector re- 


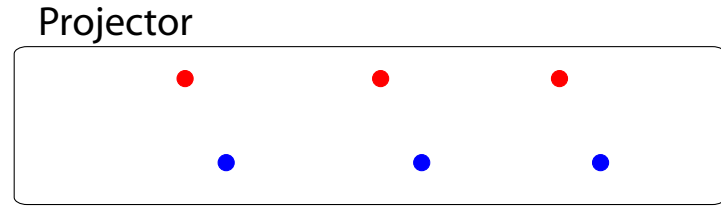

Camera

(a)
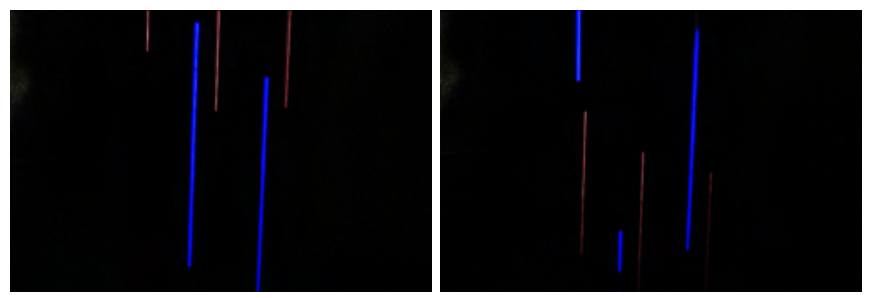

(b)
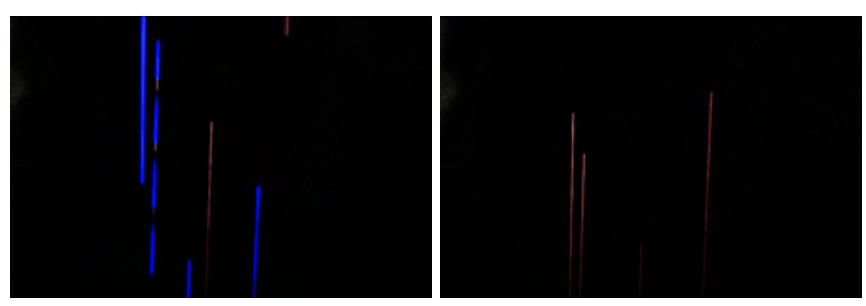

(c)

Figure 7. (a) A diagram of the two occluded layers of streaks. (b) When illuminated correctly, the streaks alternate blue and red. (c) However, when drops are too near each other, some parts of the streaks are colored incorrectly.

fresh frequency is $60 \mathrm{hz}$, meaning that we cannot handle two drops in the same location within 1/60 seconds of each other. If high drop density is required, then the method of the previous section should be used. If less-constrained drop generator geometry is required, then real-time tracking and prediction should be used.

\section{Conclusion and future work}

We have presented a method for simple calibration and design of a three-dimensional water display, using a camera, projector, and laser plane. We demonstrate examples both when drops do not occlude each other, and when they occlude each other from the point of view of the projector.

The strength of our method is that it is effective even with simple drop generators. However, because the drops came out at poorly controlled locations and times, the display had uneven density in time and space. This limits the creative possibilities. For future work, we will seek to improve control of the drops without sacrificing elegance or simplicity of design.

\section{Acknowledgments}

This research was supported in parts by Denso, ONR award N00014-08-1-0330, DURIP award N00014-06-10762 and NSF CAREER IIS-0643628. The authors would also like to thank Yaser Sheikh for discussions on modeling dynamical systems.

\section{References}

[1] T. Araki, F. Kawamata, M. Ogino, H. Miyagawa, T. Kamata, M. Watanabe, and K. Miyashita. US Patent 5,067,653: Screen forming apparatus and method. 1991.

[2] K. V. Beard. Terminal velocity and shape of cloud and precipitation drops aloft. J. of the Atmospheric Sciences, 33(5):851-864, 1976.

[3] J. Bruce, T. Balch, and M. Veloso. Fast and inexpensive color image segmentation for interactive robots. In IROS, 2000.

[4] S. Eitoku, T. Tanikawa, and Y. Suzuki. Display composed of water drops for filling space with materialized virtual threedimensional objects. In Virtual Reality Conf., 2006.

[5] G. B. Foote and P. S. duToit. Terminal velocity of raindrops aloft. J. of Applied Meteorology, 8(2):249-53, 1969.

[6] K. Garg and S. K. Nayar. Detection and removal of rain from videos. In $C V P R, 2004$.

[7] J. M. Heiner, S. E. Hudson, and K. Tanaka. The information percolator: Ambient information display in a decorative object. In Symposium on User Interface Software and Technology, 1999.

[8] M. Löffler-Mang and J. Joss. An optical disdrometer for measuring size and velocity of hydrometeors. J. of Atmospheric and Oceanic Technology, 17(2):130-139, 2000.

[9] A. V. Moere. Beyond the tyranny of the pixel: Exploring the physicality of information visualization. In Information Visualisation, 2008.

[10] J. L. Muerle and D. C. Allen. Experimental evaluation of techniques for automatic segmentation of objects in a complex scene. In Pictorial Pattern Recognition, 1968.

[11] NAO Design. infernoptix - Digital Pyrotechnic Matrix. www.infernoptix.com.

[12] K. Palovuori and I. Rakkolainen. US Patent 6,819,487: Method and apparatus for forming a projection screen or a projection volume.

[13] S. H. Pevnick. US Patent 4,294,406: Program controllable free falling water drop fountain. 1981.

[14] H. R. Pruppacher and J. D. Klett. Microphysics of Clouds and Precipitation. Kluwer Academic Publishers, second revised and enlarged edition, 1997.

[15] J. Rayner. Aquascript - information waterfall. 2007.

[16] M. Schönhuber, H. Urban, J. P. Baptista, W. Randeu, and W. Riedler. Measurements of precipitation characteristics by a new distrometer. In Conf. on Atmospheric Physics and Dynamics in the Analysis and Prognosis of Precipitation Fields, 1994. 